\title{
Double inclusive small- $x$ gluon production and their azimuthal correlations in a biased ensemble
}

\author{
Gary Kapilevich॰* \\ The Graduate School and University Center, The City University of New York, \\ 365 Fifth Avenue, New York, New York 10016, USA
}

(Received 27 November 2019; accepted 10 February 2020; published 28 February 2020)

\begin{abstract}
We consider double $g g \rightarrow g$ production in the presence of a bias on the unintegrated gluon distribution of the colliding hadrons or nuclei. Such bias could be due to the selection of configurations with a greater number of gluons or higher mean transverse momentum squared or, more generally, due to a modified spectral shape of the gluon distribution in the hadrons. Hence, we consider reweighted functional averages over the stochastic ensemble of small- $x$ gluons. We evaluate explicitly the double inclusive gluon transverse momentum spectrum in high-energy collisions, and their azimuthal correlations, for a few simple examples of biases.
\end{abstract}

DOI: $10.1103 /$ PhysRevD.101.034035

\section{INTRODUCTION}

Observables in particle collision experiments are often evaluated in biased event ensembles. For example, one may consider a class of events with greater than average multiplicity of produced particles, or events with high transverse energy deposition in a particular rapidity window, and so on. The modification of observables in such event ensembles, as compared to the minimum bias ensemble, provides insight into particle production in QCD. More generally, one could construct event ensembles using a functional bias. For example, one can define a biased event ensemble "labeled" by a prescribed function $\zeta(\vec{k}, y),{ }^{1}$ such that event $i$ is assigned a weight

$$
w_{i}=\exp \left(\int \frac{\mathrm{d}^{2} k}{(2 \pi)^{2}} \mathrm{~d} y \zeta(\vec{k}, y) \frac{\mathrm{d} N_{i}}{\mathrm{~d}^{2} k \mathrm{~d} y} / \frac{\mathrm{d} N_{\mathrm{mb}}}{\mathrm{d}^{2} k \mathrm{~d} y}\right)
$$

Here, $\frac{d N_{i}}{\mathrm{~d}^{2} k \mathrm{~d} y}$ refers to the transverse momentum distribution of particles in event $i$, and $\frac{d N_{\mathrm{mb}}}{\mathrm{d}^{2} k \mathrm{~d} y}$ is the (unweighted) average over all events. One would then study how weighted averages,

\footnotetext{
*gkapilevich@gradcenter.cuny.edu

${ }^{1}$ The function $\zeta(\vec{k})$ corresponds to $\frac{1}{2} N_{c}^{2} A_{\perp}\left(1-\eta^{-1}(\vec{k})\right)$, in terms of the function $\eta(\vec{k})$ introduced in Eqs. (14), (18) below; it is proportional to the transverse area of the collision and to the dimension of the adjoint representation.

Published by the American Physical Society under the terms of the Creative Commons Attribution 4.0 International license. Further distribution of this work must maintain attribution to the author(s) and the published article's title, journal citation, and DOI. Funded by SCOAP ${ }^{3}$.
}

$$
\langle O\rangle=\frac{\sum_{i} w_{i} O_{i}}{\sum_{i} w_{i}}
$$

of various observables depend on the bias constructed via $\zeta(\vec{k}, y)$. In this paper, we relate expectation values in biased ensembles in high-energy collisions to reweighted averages over the field configurations of small- $x$ gluons in the colliding particles. Specifically, we shall be interested in the double inclusive gluon distribution and their azimuthal correlations.

Observables in high-energy scattering in QCD are computed by expressing them in terms of expectation values of various Wilson line operators $O$; see, e.g., ref. [1]. The expectation value $\langle O\rangle$ corresponds to a statistical average [2] over the distribution of small- $x$ gluon fields. Hence, the Wilson lines from which $O$ is constructed are computed in the soft gluon field sourced by the valence color charge density $\rho$, which is the large component of the light-cone color current due to the partons with large lightcone momenta [3]:

$-\nabla_{\perp}^{2} \int d x^{-} A^{+a}\left(x^{-}, \vec{x}_{\perp}\right) \equiv-\nabla_{\perp}^{2} A^{+a}\left(\vec{x}_{\perp}\right)=g \rho^{a}\left(\vec{x}_{\perp}\right)$.

We have assumed that the fast hadron propagates in the positive $z$ direction, and that $\rho\left(\vec{x}_{\perp}\right)$ is the source in covariant gauge. We may also compute the average leading twist (covariant gauge) gluon distribution itself, via

$$
\left\langle g^{2} \operatorname{tr} A^{+}(\vec{k}) A^{+}(-\vec{k})\right\rangle=\int \mathcal{D} \rho W[\rho] \frac{g^{4}}{k^{4}} \operatorname{tr} \rho(\vec{k}) \rho(-\vec{k}) .
$$

The weight functional is assumed to be normalized to $\int \mathcal{D} \rho W[\rho]=1$. 
The constraint effective potential for

$$
X(\vec{k}) \equiv g^{2} \operatorname{tr} A^{+}(\vec{k}) A^{+}(-\vec{k})
$$

is given by [4]

$$
e^{-V_{\text {eff }}[X]}=\int \mathcal{D} \rho \delta\left(X(\vec{k})-\frac{g^{4}}{k^{4}} \operatorname{tr} \rho(\vec{k}) \rho(-\vec{k})\right) W[\rho] .
$$

This integrates out fluctuations of $\rho$ which do not affect the covariant gauge gluon distribution. The most likely gluon distribution from Eq. (4) can then be obtained (at leading power in $N_{c}$ ) as the stationary point of the effective potential:

$$
\frac{\delta V_{\mathrm{eff}}[X]}{\delta X(\vec{q})}=0 \rightarrow X_{s}(\vec{q})
$$

Given an observable which is a functional of $X(\vec{q})$, the ensemble average now reads

$$
\langle O[X]\rangle=\int \mathcal{D} X e^{-V_{\text {eff }}[X]} O[X] .
$$

For a Gaussian color charge density weight functional $W[\rho]$, one has [4]

$V_{\mathrm{eff}}[X(\vec{k})]=\int \frac{\mathrm{d}^{2} k}{(2 \pi)^{2}}\left[\frac{k^{4}}{g^{4} \mu^{2}(k)} X(\vec{k})-\frac{1}{2} A_{\perp} N_{c}^{2} \log X(\vec{k})\right]$,

and

$$
X_{s}(k)=\frac{1}{2} N_{c}^{2} A_{\perp} \frac{g^{4} \mu^{2}(k)}{k^{4}},
$$

where $A_{\perp}$ denotes the transverse area over which the gluon distribution has been integrated over. The function $\mu^{2}(k)$ parametrizes the Gaussian ensemble for the color charge density: $W[\rho] \sim \exp \left[-\int \mathrm{d}^{2} k /(2 \pi)^{2} \rho^{a}(\vec{k}) \rho^{a}(-\vec{k}) / 2 \mu^{2}(\vec{k})\right]$. However, the corresponding effective potential for $X(\vec{k})$ is not quadratic but of "linear minus log" form. ${ }^{2}$

To probe configurations away from the peak of the distribution, it is standard in statistical physics to compute biased (or reweighted) expectation values:

$$
\langle\mathcal{O}\rangle_{b}=\int \mathcal{D} \rho W[\rho] b[\rho] \mathcal{O}[\rho] .
$$

Just like $W[\rho]$, the bias $b[\rho]$ in general is supposed to be a gauge invariant functional of the color charge density.

\footnotetext{
${ }^{2}$ By a field redefinition, $V_{\text {eff }}[X(\vec{k})]$ can be rewritten as a Liouville potential for $\phi(\vec{k})=\log X(\vec{k}) / X_{s}(k)$; see Ref. [4].
}

Here, we impose the bias directly on the gluon distribution $X(\vec{k})$ :

$$
V_{\mathrm{eff}}[X(\vec{k})] \rightarrow V_{\mathrm{eff}}[X(\vec{k})]-\log b[X(\vec{k})],
$$

$\int \mathcal{D} X e^{-V_{\text {eff }}[X(k)]} O[X] \rightarrow \int \mathcal{D} X e^{-V_{\text {eff }}[X(k)]} b[X] O[X]$.

In particular, we choose $b[X]$ so that the most likely gluon distribution in the reweighted ensemble is shifted to

$$
X_{s, b}(\vec{k})=\eta(\vec{k}) X_{s}(k)
$$

where $\eta(\vec{k}) \geq 0$ is some prescribed function of transverse momentum. ${ }^{3}$ Defining

$$
b[X] \equiv \exp \left(\int \frac{\mathrm{d}^{2} \vec{k}}{(2 \pi)^{2}} t(\vec{k}) X(\vec{k})\right)
$$

this is achieved via

$$
t(\vec{q})=\left.(2 \pi)^{2} \frac{\delta V_{\mathrm{eff}}[X]}{\delta X(\vec{q})}\right|_{X(\vec{q})=\eta(\vec{q}) X_{s}\left(q^{2}\right)} .
$$

In fact, $b[X]$ is nothing but the generating functional for the moments of $X(\vec{k})$,

$$
Z[t]=\int \mathcal{D} X e^{-V_{\text {eff }}[X]+\log b[X]},
$$

$\left.\frac{1}{Z[t]} \frac{\delta^{n} Z[t]}{\delta t\left(\vec{k}_{1}\right) \cdots \delta t\left(\vec{k}_{n}\right)}\right|_{t \equiv 0}=\left\langle X\left(\vec{k}_{1}\right) \cdots X\left(\vec{k}_{n}\right)\right\rangle$,

while $\log b[X]$ is the cumulant generating functional.

In principle, the gluon distribution function depends on both the transverse momentum and the rapidity, $y$. It is straightforward to generalize the above to rapidity dependent biases by writing $X(\vec{q}, y)=\eta(\vec{q}, y) X_{s}(q, y)$, so that $t(\vec{q}, y)$ also depends on rapidity via Eq. (16). One could then, for example, reweight towards rare evolution trajectories. However, in this paper we only consider the McLerran-Venugopalan (MV) model [3] effective theory of color charge density fluctuations, which does not exhibit a dependence on $y$.

For the Gaussian action for $\rho$, from Eqs. (9), (10) and (16), we have, explicitly,

$$
t(\vec{k})=\left(1-\frac{1}{\eta(\vec{k})}\right) \frac{k^{4}}{g^{4} \mu^{2}(k)} .
$$

\footnotetext{
${ }^{3} \mathrm{We}$ do require that the saddle point is not shifted to a regime where the approach we described is not applicable. For example, $X_{s, b}(\vec{k})$ should not be of higher order in the coupling than $X_{s}(k)$.
} 
A particularly simple example for a gluon distribution in a biased ensemble would be

$$
\begin{aligned}
X(\vec{k}) & =\eta(\vec{k}) X_{s}\left(k^{2}\right), \\
\eta(k) & =1+\eta_{0} \Theta\left(k^{2}-\Lambda^{2}\right) \Theta\left(Q^{2}-k^{2}\right) .
\end{aligned}
$$

This simply boosts the number of gluons with transverse momenta from $\Lambda^{2}$ to $Q^{2}$ by the constant factor $1+\eta_{0}$ (one may also interpret this as a boost of the transverse momentum of the gluons by a factor of $\left.\left(1+\eta_{0}\right)^{1 / 4}\right)$. Other examples will be considered below.

To any given "distortion" $\eta(\vec{k})$, one can associate a potential, $V\left[\eta(\vec{k}) X_{s}\left(k^{2}\right)\right]$. The greater this potential, the smaller the weight of the function $X(\vec{k})=\eta(\vec{k}) X_{s}(k)$ in the ensemble average (8). Hence, a stronger bias is required to make this the dominant gluon distribution in the reweighted ensemble. Explicitly, the "penalty action" for any given $\eta(\vec{k})$ is

$$
\begin{aligned}
\Delta V_{\text {eff }}[\eta(\vec{k})] & \equiv V_{\text {eff }}[\eta(\vec{k})]-V_{\text {eff }}[\eta=1] \\
& =\frac{1}{2} N_{c}^{2} A_{\perp} \int \frac{\mathrm{d}^{2} k}{(2 \pi)^{2}}[\eta(\vec{k})-1-\log \eta(\vec{k})] .
\end{aligned}
$$

Thus, the gluon distribution $X(\vec{k})=\eta(\vec{k}) X_{s}(k)$ occurs, in the unbiased ensemble, with a probability density of $p[\eta]=\exp \left(-\Delta V_{\text {eff }}[\eta]\right)$, relative to the saddle point (in the space of functions). Note that $\eta(\vec{k})$ must be such that $\Delta V_{\text {eff }}[\eta(\vec{k})]$ is finite. Otherwise, the gluon distribution $X(\vec{k})=\eta(\vec{k}) X_{s}(k)$ is not part of the ensemble.

In the hadron or nucleus, a given $\eta(\vec{k})$ also corresponds to an excess gluon multiplicity of [4]

$$
\Delta N_{g}[\eta(\vec{k})]=\int \frac{\mathrm{d}^{2} k}{(2 \pi)^{2}} k^{2} X_{s}(k)[\eta(\vec{k})-1] .
$$

Likewise, any $\eta(\vec{k})$ can also be associated with, for example, an increased mean transverse momentum (see definition of $\left\langle k_{T}^{2}\right\rangle$ in Ref. [5], for example). We note, however, that our approach allows us to compute expectation values in an ensemble defined by a functional bias on the gluon distribution $X(\vec{k})$, rather than simply an ensemble defined with a bias on gluon number, mean transverse momentum, etc.

One may sample the gluon distributions in a biased ensemble in the form of Eq. (13) via a Metropolis algorithm. While these gluon distributions are part of the original ensemble, the standard approach of generating configurations without bias and then rejecting those that do not meet a given criteria would be prohibitive. Importance sampling with the action $V_{\text {eff }}[X]-\log b[X]$ strongly increases the overlap with the desired target ensemble. We consider the following three biases for illustration:
(1) $N_{g}$ bias corresponding to

$$
\log b[X]=N_{g}[X]=\int_{\Lambda}^{Q} \frac{\mathrm{d}^{2} k}{(2 \pi)^{2}} k^{2} X(k) .
$$

We take $\Lambda=2$ and $Q=6$; the units may be taken as $\mathrm{GeV}$, although the energy scale is arbitrary, since $b[X]$ is dimensionless. Also, we choose $A_{\perp}=10 \pi$ and $g^{4} \mu^{2}=2$ in Eq. (9). This bias does not impose a specific transverse momentum dependence on $\langle X(k)\rangle_{b}$. Rather, we let the Monte Carlo determine the optimal spectral shape.

(2) $E_{T}$ bias corresponding to

$$
\log b[X]=\frac{E_{T}[X]}{\Lambda}=\int_{\Lambda}^{Q} \frac{\mathrm{d}^{2} k}{(2 \pi)^{2} \Lambda} k^{3} X(k) .
$$

Once again, we do not impose a specific transverse momentum distribution on the gluons, and instead let the Monte Carlo determine the optimal spectral shape.

(3) $t[\eta]$ bias corresponding to

$$
\log b[X]=\int_{\Lambda}^{Q} \frac{\mathrm{d}^{2} k}{(2 \pi)^{2}} t(\vec{k}) X(\vec{k}),
$$

with $t(\vec{k})=\left(1-\eta^{-1}(k)\right) \frac{k^{4}}{g^{4} \mu^{2}}$ and the prescribed function $\eta(k)=\sqrt{k / \Lambda}$.

In all cases the unbiased ensemble is taken to be the MV model with constant $\mu^{2}$.

Figure 1 shows the results. Not surprisingly, the $N_{g}$ bias adds gluons mostly near $\Lambda$, since high- $k$ gluons come with a greater penalty action. The analytic solution for this bias is $\langle X(k)\rangle_{b} / X_{s}(k)=1 /\left[1-\frac{g^{4} \mu^{2}}{k^{2}} \Theta\left(Q^{2}-k^{2}\right) \Theta\left(k^{2}-\Lambda^{2}\right)\right]$. The $E_{T}$ bias produces a harder spectrum of excess gluons, with $\langle X(k)\rangle_{b} / X_{s}(k)=1 /\left[1-\frac{g^{4} \mu^{2}}{k \Lambda} \Theta\left(Q^{2}-k^{2}\right) \Theta\left(k^{2}-\Lambda^{2}\right)\right]$. Lastly, the $t[\eta]$ bias multiplies the gluon distribution between $\Lambda$ and $Q$ by the prescribed function $\eta(k)=\sqrt{k / \Lambda}$.

In a collision of two hadrons or nuclei, one is required to average over the color charge distributions of both projectile and target,

$$
\langle\mathcal{O}\rangle=\int \mathcal{D} \rho_{p} W\left[\rho_{p}\right] \int \mathcal{D} \rho_{T} W\left[\rho_{T}\right] \mathcal{O}\left[\rho_{p}, \rho_{T}\right] .
$$

One may then bias either one or both of the ensembles as described above.

The single-inclusive gluon production cross section in a biased ensemble has been computed previously in Ref. [6]. The main purpose of the current paper is to illustrate the 


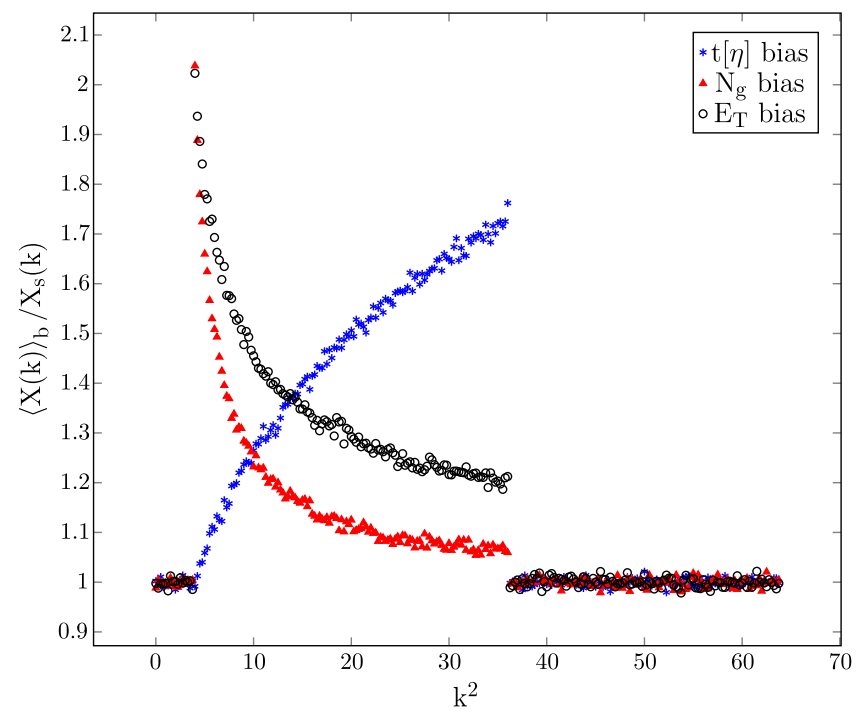

FIG. 1. Ratio of the gluon distribution in three different biased ensembles to that in the unbiased MV-model ensemble.

effect of a bias on azimuthal angular correlations of two small- $x$, high- $p_{T}$ gluons produced in a high-energy collision. We recompute the so-called "glasma graph" for a biased gluon distribution different from its expectation value $X_{s}(k)$ in the unbiased small- $x$ ensemble. These diagrams for high- $p_{T}$ double gluon production have originally been introduced in Refs. [7,8]. Their applicability, and corrections to this approximation, have been studied in Refs. [9-11].

The literature on azimuthal correlations of small- $x$ gluons is rather extensive and we do not attempt to summarize it here. Instead, we refer the reader to the review articles in Refs [12,13]. Our main focus now is on effects due to a bias on the gluon distributions of the colliding hadrons or nuclei, an issue which has rarely been addressed. A notable exception is Ref. [14], where the authors assumed that high multiplicity $p+p$ and $\mathrm{p}+\mathrm{Pb}$ events correspond to an enhanced saturation scale, $Q_{s}\left(x_{0}\right)$, of the proton at the initial rapidity for small-x evolution. The authors of Ref. [15] considered a constant multiplicative rescaling of the color charge density in the proton to discuss the multiplicity dependence of azimuthal moments [as defined in Eq. (39) below] in $\mathrm{p}+\mathrm{Pb}$ collisions. The authors of Ref. [16] analyzed angular correlations in a combinatoric model for multiparticle production with color interference effects, and their dependence on multiplicity. Finally, the authors of Ref. [17] applied a hydrodynamic model to look into the effect of final state interactions on angular correlations, as a function of the particle multiplicity in the event. Here, we perform the first analysis of "glasma graphs" in the presence of a functional bias on the gluon distribution.

The remainder of this paper is organized as follows. In Sec. II, we write the two gluon inclusive distribution at high transverse momentum for general $\eta(\vec{k})$. In Sec. III, we analyze several specific momentum dependences to see how they affect the double gluon spectrum and their angular correlations.

\section{TWO GLUON INCLUSIVE DISTRIBUTION IN A BIASED ENSEMBLE}

The cross section for inclusive production of two small- $x$ gluons with transverse momenta $p, q$ much greater than the saturation scales of the projectile and target is given by socalled glasma graphs. These graphs correspond to a $k_{T}$ factorization approximation in terms of unintegrated gluon distributions [18],

$$
\Phi(k)=\frac{1}{A_{\perp}} \frac{k^{2}}{N_{c}^{2}-1}\left\langle A^{+a}(\vec{k}) A^{+a}(-\vec{k})\right\rangle=\frac{g^{2} \mu^{2}}{k^{2}} .
$$

From now on, we consider a constant, $k$-independent $\mu^{2}$ for simplicity. This amounts to the classical MV model [3] approximation where one neglects the anomalous dimension of the gluon distribution. While it is possible, in principle, to generalize our analysis to account for the anomalous dimension due to small- $x$ evolution, our current focus is on better understanding the effect of a bias on the glasma graphs.

In a biased ensemble,

$$
\Phi_{b}(\vec{k})=\frac{g^{2} \mu^{2}}{k^{2}} \eta(\vec{k})
$$

Beyond the dilute limit, one needs to evaluate the correlator of two eikonal Wilson lines in the reweighted ensemble; see Ref. [6]. Here, we restrict our analysis to high transverse momentum, where the approximation of a dilute projectile and target should be applicable. We start with the expression for the two gluon transverse momentum distribution for glasma graphs given in Ref. $[19]^{4}$ :

$$
\begin{aligned}
\frac{\mathrm{d} N}{\mathrm{~d} y_{p} \mathrm{~d}^{2} p \mathrm{~d} y_{q} \mathrm{~d}^{2} q}= & 16 N_{c}^{2}\left(N_{c}^{2}-1\right) g^{12} \frac{A_{\perp} \Lambda^{2}}{p^{4} q^{4} \Lambda^{4}} \frac{\mu_{T}^{4} \mu_{P}^{4}}{(2 \pi)^{2}} \\
& \times(\mathcal{A}+\mathcal{B}+\mathcal{C}) .
\end{aligned}
$$

Here, $\Lambda$ denotes an infrared cutoff for applicability of the leading twist, weak field approximation. $A_{\perp} \Lambda^{2}$ will be taken to be $\sim 1$ or greater. $\mathcal{A}$ corresponds to the disconnected diagram for inclusive double gluon production shown in Fig. 2; $\mathcal{C}$ are the HBT-like [20] parts proportional to $\delta^{2}(\vec{p} \pm \vec{q})$, shown in Fig. 3; and the remaining diagrams are combined into $\mathcal{B}$ (Fig. 4), and have been interpreted as Bose enhancement [21]. Note that $\mathcal{B}$ and $\mathcal{C}$ correspond to connected two gluon production diagrams.

\footnotetext{
${ }^{4}$ However, we neglect corrections due to the nonzero thickness of the projectile or target derived in Ref. [19].
} 
Explicitly,

$$
\begin{aligned}
\mu_{T}^{4} \mu_{P}^{4} g^{8} \mathcal{A} & =\frac{\left(N_{c}^{2}-1\right) A_{\perp} \Lambda^{2} p^{2} q^{2}}{(2 \pi)^{2}} \int_{\Lambda^{2}}^{\infty} \mathrm{d}^{2} k_{1} \Phi_{P}\left(\overrightarrow{k_{1}}\right) \Phi_{T}\left(\overrightarrow{k_{1}}-\vec{p}\right) \int_{\Lambda^{2}}^{\infty} \mathrm{d}^{2} k_{2} \Phi_{P}\left(\overrightarrow{k_{2}}\right) \Phi_{T}\left(\overrightarrow{k_{2}}-\vec{q}\right), \\
\mu_{T}^{4} \mu_{P}^{4} g^{8} \mathcal{B}= & \Lambda^{2} p^{2} q^{2} \int_{\Lambda^{2}}^{\infty} \mathrm{d}^{2} k \Phi_{T}(\vec{k}-\vec{p}) \Phi_{P}(\vec{k})\left[\Phi_{P}(\vec{k}) \Phi_{T}(\vec{k}-\vec{q})+\Phi_{T}(\vec{k}-\vec{p}) \Phi_{P}(\vec{k}-\vec{p}-\vec{q})\right. \\
& +\frac{1}{8} \Phi_{T}(\vec{k}-\vec{p}) \Phi_{P}(\vec{k}-\vec{p}-\vec{q}) \frac{f(\vec{k}, \vec{p}, \vec{q})}{\left.(\vec{k}-\vec{p}-\vec{q})^{2} k^{2}(\vec{k}-\vec{q})^{4}\right]+(\vec{q} \rightarrow-\vec{q}),}
\end{aligned}
$$

where

$$
f(\vec{k}, \vec{p}, \vec{q})=\left[k^{2}(\vec{k}-\vec{q})^{2}+(\vec{p}+\vec{q}-\vec{k})^{2}(\vec{p}-\vec{k})^{2}-p^{2}(2 \vec{k}-\vec{q}-\vec{p})^{2}\right]\left[(\vec{p}+\vec{q}-\vec{k})^{2}(\vec{k}-\vec{q})^{2}+k^{2}(\vec{p}-\vec{k})^{2}-q^{2}(2 \vec{k}-\vec{q}-\vec{p})^{2}\right] .
$$

The contribution from HBT diagrams is

$$
\begin{aligned}
\mu_{T}^{4} \mu_{P}^{4} g^{8} \mathcal{C}= & \frac{\Lambda^{2} p^{2} q^{2}}{4} \int_{\Lambda^{2}}^{\infty} \mathrm{d}^{2} k_{1} \mathrm{~d}^{2} k_{2} \Phi_{P}\left(\overrightarrow{k_{1}}\right) \Phi_{P}\left(\overrightarrow{k_{2}}\right) \Phi_{T}\left(\overrightarrow{k_{1}}-\vec{p}\right) \Phi_{T}\left(\overrightarrow{k_{2}}-\vec{q}\right) \\
& \times\left[\delta^{2}(\vec{p}-\vec{q})\left\{1+\frac{k_{2}^{2}\left(\vec{p}-\overrightarrow{k_{1}}\right)^{2}}{k_{1}^{2}\left(\vec{p}-\overrightarrow{k_{2}}\right)^{2}}-\frac{p^{2}\left(\overrightarrow{k_{1}}-\overrightarrow{k_{2}}\right)^{2}}{k_{1}^{2}\left(\vec{p}-\overrightarrow{k_{2}}\right)^{2}}\right\}\left\{1+\frac{k_{1}^{2}\left(\vec{q}-\overrightarrow{k_{2}}\right)^{2}}{k_{2}^{2}\left(\vec{q}-\overrightarrow{k_{1}}\right)^{2}}-\frac{q^{2}\left(\overrightarrow{k_{1}}-\overrightarrow{k_{2}}\right)^{2}}{k_{2}^{2}\left(\vec{q}-\overrightarrow{k_{1}}\right)^{2}}\right\}\right. \\
& \left.+\delta^{2}(\vec{p}+\vec{q})\left\{1+\frac{k_{2}^{2}\left(\vec{p}-\overrightarrow{k_{1}}\right)^{2}}{k_{1}^{2}\left(\vec{p}+\overrightarrow{k_{2}}\right)^{2}}-\frac{p^{2}\left(\overrightarrow{k_{1}}+\overrightarrow{k_{2}}\right)^{2}}{k_{1}^{2}\left(\vec{p}+\overrightarrow{k_{2}}\right)^{2}}\right\}\left\{1+\frac{k_{1}^{2}\left(\vec{q}-\overrightarrow{k_{2}}\right)^{2}}{k_{2}^{2}\left(\vec{q}+\overrightarrow{k_{1}}\right)^{2}}-\frac{q^{2}\left(\overrightarrow{k_{1}}+\overrightarrow{k_{2}}\right)^{2}}{k_{2}^{2}\left(\vec{q}+\overrightarrow{k_{1}}\right)^{2}}\right\}\right] .
\end{aligned}
$$

Note that contributions $\mathcal{B}$ and $\mathcal{C}$, arising from connected diagrams, come with neither a second power of the transverse area, $A_{\perp}$, nor a second factor of $N_{c}^{2}-1$. This is because there is a single connected color flow loop.

It is sufficient for our present purposes to consider a bias on the target's ensemble of gluon distributions only. We first compute the contributions denoted by $\mathcal{A}$ and $\mathcal{B}$. We will compute these assuming that $\vec{p} \neq \pm \vec{q}$, i.e., that $\vec{p} \pm \vec{q}$ are hard momenta themselves, much greater than the saturation scales of the colliding protons or nuclei. In fact, when the magnitudes of $\vec{p}$ and $\vec{q}$ are close, one needs to also account for the back-to-back dijet contribution (see e.g., Ref. [14]) when computing angular correlations of high- $p_{T}$ gluons. Therefore, while we do give the expression for $\mathcal{C}$ for general $\eta(\vec{k})$ later in this section for completeness, we do not consider these contributions further in Sec. III.

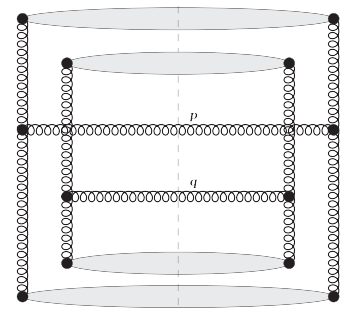

FIG. 2. Disconnected diagram for inclusive production of two gluons with momenta $p$ and $q$.
In this paper, we will only consider reflection symmetric gluon distributions: $\eta(-\vec{p})=\eta(\vec{p})$. To perform the integrations over the transverse momenta of the gluons in projectile and target, we expand the integrands in Eqs. (29), (30), (32) around the singularities of the Coulomb propagators, and keep the leading terms. For example,

$$
\int_{\Lambda^{2}} \frac{\mathrm{d}^{2} k}{k^{2}} \frac{\eta(\vec{k}-\vec{p})}{(\vec{k}-\vec{p})^{2}}=\frac{2 \pi}{p^{2}} \log \frac{p^{2}}{\Lambda^{2}}+\int_{\Lambda^{2}} \frac{\mathrm{d}^{2} k}{k^{2}} \frac{\eta(\vec{k}-\vec{p})-1}{(\vec{k}-\vec{p})^{2}} .
$$

The first term is the DGLAP logarithm [22]. To compute the integral in the second term, we first write $\eta(\vec{k})-1=$ $\tilde{\eta}(k) \Theta\left(Q^{2}-k^{2}\right)$ to display explicitly the finite support of the modification to the gluon distribution. Now, if $Q^{2}$ is on the order of $p^{2}$, the contribution from small $k^{2} \ll p^{2}$ to the integral is
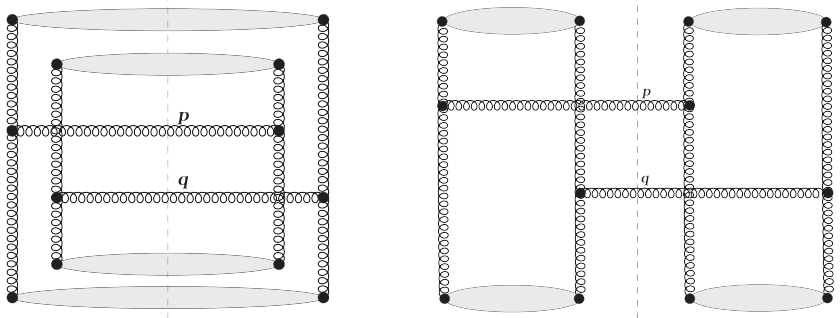

FIG. 3. HBT diagrams proportional to $\delta(\vec{p} \pm \vec{q})$. 

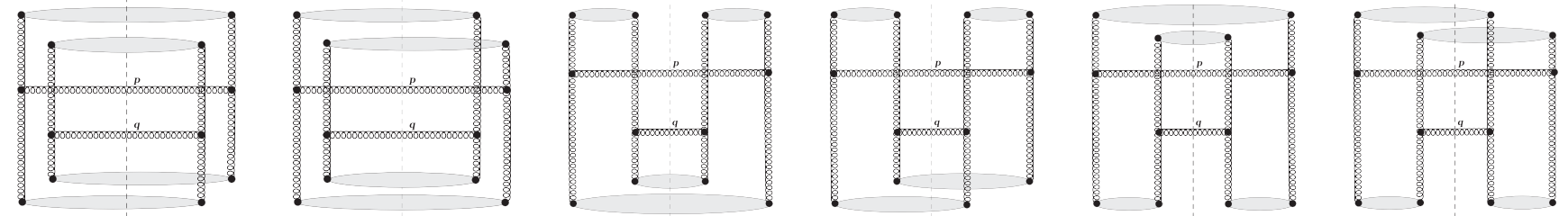

FIG. 4. Bose enhancement (B-type) diagrams.

$$
I_{1}(\vec{p})=\frac{\pi}{p^{2}} \tilde{\eta}(\vec{p}) \log \frac{p^{2}}{\Lambda^{2}}, \quad\left(\text { if } Q^{2} \sim p^{2}\right)
$$

This contribution is absent ${ }^{5}$ if $Q^{2} \ll p^{2}$. For any $Q^{2} \sim p^{2}$ or less, the integral on the right-hand side of Eq. (33) also receives a contribution from the region $(\vec{p}-\vec{k})^{2} \ll p^{2}$, provided that $\tilde{\eta}(\vec{\ell}) / \ell^{2}$ has a pole at $\ell \rightarrow 0$ :

$$
\begin{aligned}
I_{2}(\vec{p})= & \frac{1}{p^{2}} \int_{\Lambda^{2}}^{Q^{2}} \frac{\mathrm{d}^{2} \ell}{\ell^{2}} \tilde{\eta}(\vec{\ell}), \\
& \left(\text { if } \tilde{\eta}(\vec{\ell}) / \ell^{2} \text { has a pole at } \ell \rightarrow 0\right) .
\end{aligned}
$$

Then Eq. (29) is approximated as

$$
\mathcal{A} \approx \frac{\left(N_{c}^{2}-1\right) A_{\perp} \Lambda^{2}}{(2 \pi)^{2}}\left[2 \pi \log \frac{p^{2}}{\Lambda^{2}}+p^{2} I_{1}(\vec{p})+p^{2} I_{2}(\vec{p})\right]\left[2 \pi \log \frac{q^{2}}{\Lambda^{2}}+q^{2} I_{1}(\vec{q})+q^{2} I_{2}(\vec{q})\right] .
$$

Of course, in an unbiased ensemble where $\eta(\vec{k})-1=\tilde{\eta}(k)=0$, this contribution for independent production of two gluons does not depend on the angle between $\vec{p}$ and $\vec{q}$. The same is true if $\eta(\vec{k})$ is isotropic.

For the contribution from connected two-gluon production diagrams we obtain

$$
\begin{aligned}
\mathcal{B} \approx & \left\{\frac{\Lambda^{2}}{(\vec{p}+\vec{q})^{2}}\left[\frac{q^{2}}{p^{2}} \int^{\min \left(p^{2},(\vec{p}+\vec{q})^{2}\right)} \frac{\mathrm{d}^{2} k}{k^{2}}\left[\eta(\vec{k}) \eta(\vec{p}+\vec{q})+\eta^{2}(\vec{p})\right]+(\vec{p} \leftrightarrow \vec{q})\right]+\frac{1}{2} \frac{\Lambda^{2}}{p^{2} q^{2}(\vec{p}+\vec{q})^{4}}\right. \\
& \left.\times \int_{\min \left(p^{2}, q^{2},(\vec{p}+\vec{q})^{2}\right)} \frac{\mathrm{d}^{2} k}{k^{4}} g(\vec{k}, \vec{p}, \vec{q})\left[\eta^{2}(\vec{p})+\eta^{2}(\vec{q})+\eta^{2}(\vec{p}+\vec{q})+\eta^{2}(\vec{k})\right]+\frac{\Lambda^{2}}{2} \int^{\min \left(p^{2}, q^{2}\right)} \mathrm{d}^{2} k \frac{\eta^{2}(\vec{k})}{k^{4}}\right\}+(\vec{q} \rightarrow-\vec{q}) .
\end{aligned}
$$

Here,

$$
g(\vec{k}, \vec{p}, \vec{q})=\left[p^{2} \vec{k} \cdot(\vec{p}+\vec{q})-(\vec{p}+\vec{q})^{2} \vec{k} \cdot \vec{p}\right]\left[q^{2} \vec{k} \cdot(\vec{p}+\vec{q})-(\vec{p}+\vec{q})^{2} \vec{k} \cdot \vec{q}\right]
$$

is one-fourth the leading term of $f(\vec{k}, \vec{p}, \vec{q})$ in the limit $k^{2} \ll p^{2}, q^{2}$.

The expansion in Eq. (37) includes terms that explicitly depend on the azimuthal angle, $\phi$, between $\vec{p}$ and $\vec{q}$, even though they may be subleading at large $p^{2}, q^{2}$. In contrast, we have dropped a term in Eq. (37) that does not depend on $\phi$, and that would be subleading when $\mathcal{A}$ and $\mathcal{B}$ are added. However, we have not dropped the last term in Eq. (37), which exhibits power sensitivity to low transverse momenta but is independent of $\phi$ when $\eta(\vec{k})$ is isotropic. In Sec. III we will compute the angular moments

\footnotetext{
${ }^{5}$ To smoothly interpolate from $Q^{2} \sim \Lambda^{2}$ to $Q^{2} \sim p^{2}$, one could replace the logarithm in Eq. (34) by $\log p^{2} /\left(p^{2}-Q^{2}+\Lambda^{2}\right)$. However, we prefer to avoid such ad hoc interpolations, and instead distinguish small and large $Q^{2}$ explicitly.
}

$$
\left\langle e^{i n \phi}\right\rangle=\frac{\int \frac{\mathrm{d} N}{\mathrm{~d} y_{p} \mathrm{~d}^{2} p \mathrm{~d} y_{q} \mathrm{~d}^{2} q} e^{i n \phi} \mathrm{d} \phi}{\int \frac{\mathrm{d} N}{\mathrm{~d} y_{p} \mathrm{~d}^{2} p \mathrm{~d} y_{q} \mathrm{~d}^{2} q} \mathrm{~d} \phi} \rightarrow \frac{\int \frac{\mathrm{d} N}{\mathrm{~d} y_{p} \mathrm{~d}^{2} p \mathrm{~d}_{q} \mathrm{~d}^{2} q} \cos (n \phi) \mathrm{d} \phi}{\int \frac{\mathrm{d} N}{\mathrm{~d} y_{p} \mathrm{~d}^{2} p \mathrm{~d}_{q} \mathrm{~d}^{2} q} \mathrm{~d} \phi} .
$$

Reflection symmetry under the simultaneous $\vec{p} \rightarrow-\vec{p}, \vec{q} \rightarrow$ $-\vec{q}$ implies invariance under $\phi \rightarrow-\phi$, and so $\left\langle e^{i n \phi}\right\rangle$ is real.

If $\eta(\vec{k})$ is isotropic, Eq. (37) gives the leading $\phi$-dependent terms. However, in Sec. III D we shall see that, when $\eta(\vec{k})$ is anisotropic, Eq. (36) will also contribute to the angular moments. In that case, the angular correlations in the "disconnected diagrams" actually arise due to the bias.

Using the same approximation for the integrations over the $2 \mathrm{~d}$ Coulomb propagators, the "HBT diagrams" for general $\eta(\vec{k})$ evaluate to 


$$
\begin{aligned}
\mathcal{C} \approx & \Lambda^{2}\left[\delta^{2}(\vec{p}-\vec{q})+\delta^{2}(\vec{p}+\vec{q})\right]\left\{\frac{\pi^{2}}{2} \eta^{2}(\vec{p}) \log ^{2} \frac{p^{2}}{\Lambda^{2}}\right. \\
& +\int_{\Lambda^{2}}^{p^{2}} \frac{\mathrm{d}^{2} k_{1}}{k_{1}^{4}} \frac{\mathrm{d}^{2} k_{2}}{k_{2}^{4}} \eta\left(\vec{k}_{1}\right)\left[\left(\vec{k}_{1} \cdot \vec{k}_{2}\right)^{2} \eta\left(\vec{k}_{2}\right)+2 \eta(\vec{p})\right. \\
& \left.\left.\times\left(\vec{k}_{1} \cdot \vec{k}_{2}-\frac{2\left(\vec{k}_{1} \cdot \vec{p}\right)\left(\vec{k}_{2} \cdot \vec{p}\right)}{p^{2}}\right)^{2}\right]\right\} .
\end{aligned}
$$

\section{SPECIFIC ENSEMBLES}

In this section, we evaluate explicitly the contributions from diagrams $\mathcal{A}$ and $\mathcal{B}$ for a few choices of $\eta(\vec{k})$. As already mentioned above, we will focus on the case where the transverse momenta $\vec{p}$ and $\vec{q}$ do not have very similar magnitudes, and so will ignore diagrams of type $\mathcal{C}$.

We study a biased ensemble where the number of gluons (defined from the covariant gauge gluon distribution) with squared transverse momenta between $\Lambda^{2}$ and $Q^{2}$ is boosted. In this section, we consider ensembles of the form

$\eta(\vec{k})=1+\eta_{0} \frac{\Lambda^{2 a}}{k^{2 a}}(\hat{k} \cdot \hat{E})^{2 b} \Theta\left(Q^{2}-k^{2}\right) \Theta\left(k^{2}-\Lambda^{2}\right)$,

where $\eta_{0}$ is a dimensionless constant, $a$ controls the transverse momentum dependence, and $b \geq 0$ the anisotropy (in the direction $\hat{E}$ ). We consider isotropic ensembles with $a=0$ (Sec. III B) and $a=1$ (Sec. III C), as well as anisotropic ensembles with $a=0$ and $b=1$ (Sec. III D). We will also briefly discuss the case $a=-1$, $b=0$ at the end of Sec. III C.

\section{A. Unbiased ensemble}

Setting $\eta(\vec{k})=1$ in Eqs. (36), (37), we have

$$
\begin{aligned}
{\left[\frac{\mathrm{d} N}{\mathrm{~d} y_{p} \mathrm{~d}^{2} p \mathrm{~d} y_{q} \mathrm{~d}^{2} q}\right]_{\mathrm{unb}} \approx } & 16 N_{c}^{2}\left(N_{c}^{2}-1\right) g^{12} \frac{A_{\perp}}{p^{4} q^{4} \Lambda^{2}} \frac{\mu_{T}^{4} \mu_{P}^{4}}{(2 \pi)^{2}}\left\{\frac{1}{2}\left(N_{c}^{2}-1\right)\left(A_{\perp} \Lambda^{2}\right) \log \frac{p^{2}}{\Lambda^{2}} \log \frac{q^{2}}{\Lambda^{2}}+\mathcal{O}(1)\right. \\
& +2 \pi \frac{\Lambda^{2}}{(\vec{q}+\vec{p})^{2}}\left[\frac{q^{2}}{p^{2}} \log \frac{\min \left(p^{2},(\vec{q}+\vec{p})^{2}\right)}{\Lambda^{2}}+\frac{p^{2}}{q^{2}} \log \frac{\min \left(q^{2},(\vec{q}+\vec{p})^{2}\right)}{\Lambda^{2}}\right. \\
& \left.\left.-\frac{1}{2}\left(1+\vec{q} \cdot \vec{p}\left(\frac{1}{p^{2}}+\frac{1}{q^{2}}\right)\right) \log \frac{\min \left(p^{2}, q^{2},(\vec{q}+\vec{p})^{2}\right)}{\Lambda^{2}}\right]+\mathcal{O}\left(\frac{\Lambda^{2}}{p^{2}}\right)\right\}+(\vec{q} \rightarrow-\vec{q}) .
\end{aligned}
$$

Here, $\mathcal{O}(1)$ stands for the subleading $\phi$-independent terms, while $\mathcal{O}\left(\frac{\Lambda^{2}}{p^{2}}\right)$ stands for the subleading $\phi$-dependent terms. Only the $\phi$-dependent terms enter into the numerator of Eq. (39), from which we can see that the odd moments vanish. To compute the even moments, we will need the integrals

$$
\begin{gathered}
\int \mathrm{d} \phi \cos (2 n \phi)\left(\frac{1}{(\vec{p}-\vec{q})^{2}}+\frac{1}{(\vec{p}+\vec{q})^{2}}\right)=\frac{4 \pi}{\left|p^{2}-q^{2}\right|} \frac{q_{<}^{2 n}}{q_{>}^{2 n}} \\
\int \mathrm{d} \phi \cos (2 n \phi)\left(\frac{\vec{q} \cdot \vec{p}}{(\vec{p}-\vec{q})^{2}}-\frac{\vec{q} \cdot \vec{p}}{(\vec{p}+\vec{q})^{2}}\right)=2 \pi \frac{p^{2}+q^{2}}{\left|p^{2}-q^{2}\right|} \frac{q_{<}^{2 n}}{q_{>}^{2 n}},
\end{gathered}
$$

which can be derived with contour integration. Here $q_{<}^{2}=\min \left(p^{2}, q^{2}\right)$ and $q_{>}^{2}=\max \left(p^{2}, q^{2}\right)$. To use Eqs. (43), (44) in (42), we need to neglect the dependence of $(\vec{p} \pm \vec{q})^{2}$ on $\phi$ when the former appears inside a logarithm. This is justified in leading logarithmic approximation. The even angular moments for $n \geq 1$ then read

$$
\left\langle e^{2 n i \phi}\right\rangle_{\mathrm{unb}} \approx \frac{\pi}{\left(N_{c}^{2}-1\right) A_{\perp} \Lambda^{2}} \frac{\Lambda^{2}}{\left|p^{2}-q^{2}\right|} \frac{q_{<}^{2 n}}{q_{>}^{2 n}} \frac{\left(\frac{q^{2}}{p^{2}}+\frac{p^{2}}{q^{2}}\right) \log \frac{\min \left(p^{2}, q^{2}, \ell^{2}\right)}{\Lambda^{2}}+4 \frac{q^{2}}{p^{2}} \log \frac{\min \left(p^{2}, \ell^{2}\right)}{\Lambda^{2}}+4 \frac{p^{2}}{q^{2}} \log \frac{\min \left(q^{2}, \ell^{2}\right)}{\Lambda^{2}}}{\log \frac{p^{2}}{\Lambda^{2}} \log \frac{q^{2}}{\Lambda^{2}}},
$$

where $\ell^{2}=p^{2}+q^{2}-2 p q$. This formula predicts that $\left\langle e^{2 n i \phi}\right\rangle$ decreases with $n$ like $\left[\min \left(p^{2}, q^{2}\right) / \max \left(p^{2}, q^{2}\right)\right]^{n}$. When ${ }^{6}$ $p^{2} \approx q^{2}$, Eq. (45) simplifies to

$$
\left\langle e^{2 n i \phi}\right\rangle_{\mathrm{unb}} \approx \frac{10 \pi}{\left(N_{c}^{2}-1\right) A_{\perp} \Lambda^{2}} \frac{\Lambda^{2}}{\left|p^{2}-q^{2}\right|} \frac{\log \frac{\ell^{2}}{\Lambda^{2}}}{\log \frac{p^{2}}{\Lambda^{2}} \log \frac{q^{2}}{\Lambda^{2}}}
$$

\footnotetext{
${ }^{6}$ As explained in Sec. II, we do not consider the case where $p$ and $q$ are very similar. What we mean here is that their difference should be less than $p$ and $q$ themselves (but still much greater than the saturation scales of projectile and target).
} 


\section{B. Constant boost of the gluon density between $k^{2}=\Lambda^{2}$ and $k^{2}=Q^{2}$}

In this section, we consider a boost of the gluon density between $k^{2}=\Lambda^{2}$ and $k^{2}=Q^{2}$ by a constant factor $1+\eta_{0}$ :

$$
\eta(\vec{k})=1+\eta_{0} \Theta\left(Q^{2}-k^{2}\right) \Theta\left(k^{2}-\Lambda^{2}\right) .
$$

This corresponds to the "penalty" action

$$
V_{\mathrm{eff}}=\frac{1}{8 \pi} N_{c}^{2} A_{\perp} \Lambda^{2} \frac{Q^{2}-\Lambda^{2}}{\Lambda^{2}}\left[\eta_{0}-\log \left(1+\eta_{0}\right)\right],
$$

and to a gluon number excess

$$
\Delta N_{g}=\frac{1}{8 \pi} N_{c}^{2} A_{\perp} g^{4} \mu^{2} \eta_{0} \log \frac{Q^{2}}{\Lambda^{2}} .
$$

Hence, for $\eta(k)$ like in Eq. (47), a substantial gluon number excess is much more likely to occur due to many additional gluons with small transverse momenta not too far above $\Lambda$, so that $\eta_{0}$ is large but $Q^{2} / \Lambda^{2}$ is moderate. However, such configurations do not increase $\left\langle k_{T}^{2}\right\rangle$ much [biasing towards gluon distributions like Eq. (47) with large $\left\langle k_{T}^{2}\right\rangle$ would rather favor smaller $\eta_{0}$ and larger $Q^{2}$ ].

We first calculate the two-gluon spectrum for $Q^{2} \sim$ $p^{2}, q^{2},(\vec{p} \pm \vec{q})^{2}$. Factoring $\left(1+\eta_{0}\right)^{2}$ from Eqs. (29) and (30), we have

$$
\left[\frac{\mathrm{d} N}{\mathrm{~d} y_{p} \mathrm{~d}^{2} p \mathrm{~d} y_{q} \mathrm{~d}^{2} q}\right]_{\text {bias }}=\left(1+\eta_{0}\right)^{2}\left[\frac{\mathrm{d} N}{\mathrm{~d} y_{p} \mathrm{~d}^{2} p \mathrm{~d} y_{q} \mathrm{~d}^{2} q}\right]_{\text {unb }} .
$$

We conclude that, in the limit where $Q^{2}$ is on the order of the momenta of the produced gluons (or greater), the angular moments in this ensemble are the same as in the unbiased ensemble, cf. Eq. (45). This is analogous to the $k$-independent rescaling of the color charge density of the proton considered in Ref. [15]. Note, however, that such gluon distributions have very small probability in the original ensemble, since $V_{\text {eff }} \propto Q^{2}$.

On the other hand, when $Q^{2} \ll p^{2}, q^{2},(\vec{p} \pm \vec{q})^{2}$, we can drop contributions like those in Eq. (34). Then

$$
\begin{aligned}
{\left[\frac{\mathrm{d} N}{\mathrm{~d} y_{p} \mathrm{~d}^{2} p \mathrm{~d} y_{q} \mathrm{~d}^{2} q}\right]_{\text {bias }} \approx } & 16 N_{c}^{2}\left(N_{c}^{2}-1\right) g^{12} \frac{A_{\perp}}{p^{4} q^{4} \Lambda^{2}} \frac{\mu_{T}^{4} \mu_{P}^{4}}{(2 \pi)^{2}} \\
& \times\left\{\frac{1}{2}\left(N_{c}^{2}-1\right)\left(A_{\perp} \Lambda^{2}\right)\left(\log \frac{p^{2}}{\Lambda^{2}}+\frac{\eta_{0}}{2} \log \frac{Q^{2}}{\Lambda^{2}}\right)\left(\log \frac{q^{2}}{\Lambda^{2}}+\frac{\eta_{0}}{2} \log \frac{Q^{2}}{\Lambda^{2}}\right)+\mathcal{O}(1)\right. \\
& +2 \pi \frac{\Lambda^{2}}{(\vec{q}+\vec{p})^{2}}\left[\frac{q^{2}}{p^{2}}\left(\log \frac{\min \left(p^{2},(\vec{q}+\vec{p})^{2}\right)}{\Lambda^{2}}+\frac{\eta_{0}}{2} \log \frac{Q^{2}}{\Lambda^{2}}\right)\right. \\
& +\frac{p^{2}}{q^{2}}\left(\log \frac{\min \left(q^{2},(\vec{q}+\vec{p})^{2}\right)}{\Lambda^{2}}+\frac{\eta_{0}}{2} \log \frac{Q^{2}}{\Lambda^{2}}\right) \\
& \left.-\frac{1}{2}\left(1+\vec{q} \cdot \vec{p}\left(\frac{1}{p^{2}}+\frac{1}{q^{2}}\right)\right)\left(\log \frac{\min \left(p^{2}, q^{2},(\vec{q}+\vec{p})^{2}\right)}{\Lambda^{2}}+\left(\frac{\eta_{0}}{2}+\frac{\eta_{0}^{2}}{4}\right) \log \frac{Q^{2}}{\Lambda^{2}}\right)\right] \\
& \left.+\mathcal{O}\left(\frac{\Lambda^{2}}{p^{2}}\right)\right\}+(\vec{q} \rightarrow-\vec{q}) .
\end{aligned}
$$

It may be surprising, at first glance, that a "distortion" of the gluon distribution up to $Q^{2}$ much less than the momenta of the produced gluons would affect the cross section. This is due to the fact that the production occurs via Lipatov fusion [23] of one gluon from the projectile with one gluon from the target, where typically one of the fusing gluons carries much smaller transverse momentum than the produced gluon.

The even angular moments for $n \geq 1$ resulting from Eq. (51) are

$$
\begin{aligned}
\left\langle e^{2 n i \phi}\right\rangle_{\text {bias }} \approx & \frac{\pi}{\left(N_{c}^{2}-1\right) A_{\perp} \Lambda^{2}} \frac{\Lambda^{2}}{\left|p^{2}-q^{2}\right|} \frac{q_{<}^{2 n}}{q_{>}^{2 n}} \\
& \times \frac{\left(\frac{q^{2}}{p^{2}}+\frac{p^{2}}{q^{2}}\right)\left(\log \frac{\min \left(p^{2}, q^{2}, \ell^{2}\right)}{\Lambda^{2}}+\left(\frac{5 \eta_{0}}{2}+\frac{\eta_{0}^{2}}{4}\right) \log \frac{Q^{2}}{\Lambda^{2}}\right)+4 \frac{q^{2}}{p^{2}} \log \frac{\min \left(p^{2}, \ell^{2}\right)}{\Lambda^{2}}+4 \frac{p^{2}}{q^{2}} \log \frac{\min \left(q^{2}, \ell^{2}\right)}{\Lambda^{2}}}{\left(\log \frac{p^{2}}{\Lambda^{2}}+\frac{\eta_{0}}{2} \log \frac{Q^{2}}{\Lambda^{2}}\right)\left(\log \frac{q^{2}}{\Lambda^{2}}+\frac{\eta_{0}}{2} \log \frac{Q^{2}}{\Lambda^{2}}\right)} .
\end{aligned}
$$

We recall that $\ell^{2}=p^{2}+q^{2}-2 p q$. 
We have yet to make any assumptions about the magnitude of $\eta_{0}$. First, consider $\eta_{0}=\mathcal{O}(1)$, with $Q^{2}$ less than $\min \left(p^{2}, q^{2}, \ell^{2}\right)$. This corresponds to a class of high gluon multiplicity configurations with $\left\langle k_{T}^{2}\right\rangle$ moderately higher than in the absence of the bias. Here, the corrections to the numerator and denominator of Eq. (52) due to the bias are suppressed only logarithmically (relative to the unbiased ensemble). On the other hand, if $\eta_{0} \ll 1$, we can simplify the previous expression by ignoring the terms $\sim \eta_{0}^{2}$ to compute the ratio of the moments in the biased and unbiased ensembles. ${ }^{7}$ For $\ell^{2}<\min \left(p^{2}, q^{2}\right)$, for example, this is

$$
\frac{\left\langle e^{2 n i \phi}\right\rangle_{\text {bias }}}{\left\langle e^{2 n i \phi}\right\rangle_{\text {unb }}} \approx 1+\frac{\eta_{0}}{2} \frac{\log \frac{Q^{2}}{\Lambda^{2}}}{\log \frac{\ell^{2}}{\Lambda^{2}}}\left(1-\frac{\log \frac{\ell^{2}}{\Lambda^{2}}}{\log \frac{q^{2}}{\Lambda^{2}}}-\frac{\log \frac{\ell^{2}}{\Lambda^{2}}}{\log \frac{p^{2}}{\Lambda^{2}}}\right) .
$$

Thus, when $\vec{p}$ and $\vec{q}$ have comparable magnitudes $\left(\rightarrow \ell^{2} \ll p^{2}, q^{2}\right)$, the correction is positive, and so the angular moments in the biased ensemble increase with increasing $\eta_{0}$. In the limit $p^{2} \gg q^{2}$ (or vice versa), on the other hand, the correction in Eq. (52) is negative,

$$
\frac{\left\langle e^{2 n i \phi}\right\rangle_{\text {bias }}}{\left\langle e^{2 n i \phi}\right\rangle_{\text {unb }}} \approx 1-\frac{\eta_{0}}{2} \frac{\log \frac{Q^{2}}{\Lambda^{2}}}{\log \frac{p^{2}}{\Lambda^{2}}},
$$

and so the angular moments decrease with increasing $\eta_{0}$. Thus, we see that even a very simple "distortion" of the gluon distribution may give rise to a fairly intricate behavior of the angular correlations.

\section{Transverse momentum dependent boost of the gluon density}

We now consider a transverse momentum dependent increase of the gluon density between $k^{2}=\Lambda^{2}$ and $k^{2}=Q^{2}$. We will mostly confine ourselves to an $\eta(k)$ that decreases with increasing $k$, but will briefly take up the case of $\eta(k)$ increasing with $k$ at the end of this section. Let us then start with

$$
\eta(k)=1+\eta_{0} \frac{\Lambda^{2}}{k^{2}} \Theta\left(Q^{2}-k^{2}\right) \Theta\left(k^{2}-\Lambda^{2}\right),
$$

with $Q^{2} \gg \Lambda^{2}$. Such a gluon distribution comes with a penalty action of

$$
V_{\mathrm{eff}}[\eta]=\frac{1}{8 \pi} N_{c}^{2} A_{\perp} \Lambda^{2}\left[\left(\frac{Q^{2}}{\Lambda^{2}}-\eta_{0}\right) \log \left(1+\eta_{0} \frac{\Lambda^{2}}{Q^{2}}\right)+\left(1+\eta_{0}\right) \log \left(1+\eta_{0}\right)\right] .
$$

Also, the number of high- $k_{T}$ gluons in the hadron increases by

$$
\Delta N_{g}[\eta]=\frac{1}{8 \pi} N_{c}^{2} A_{\perp} g^{4} \mu^{2} \eta_{0} .
$$

Hence, both $V_{\text {eff }}$ and $\Delta N_{g}$ approach a constant as $Q^{2} / \Lambda^{2} \gg 1$ at fixed $\eta_{0}$.

The resulting gluon production distribution is

$$
\begin{aligned}
{\left[\frac{\mathrm{d} N}{\mathrm{~d} y_{p} \mathrm{~d}^{2} p \mathrm{~d} y_{q} \mathrm{~d}^{2} q}\right]_{\text {bias }} \approx } & 16 N_{c}^{2}\left(N_{c}^{2}-1\right) g^{12} \frac{A_{\perp}}{p^{4} q^{4} \Lambda^{2}} \frac{\mu_{T}^{4} \mu_{P}^{4}}{(2 \pi)^{2}}\left\{\frac{1}{2}\left(N_{c}^{2}-1\right)\left(A_{\perp} \Lambda^{2}\right)\left(\log \frac{p^{2}}{\Lambda^{2}}+\frac{\eta_{0}}{2}\right)\left(\log \frac{q^{2}}{\Lambda^{2}}+\frac{\eta_{0}}{2}\right)+\mathcal{O}(1)\right. \\
+ & \frac{\pi}{3} \eta_{0}^{2}+2 \pi \frac{\Lambda^{2}}{(\vec{q}+\vec{p})^{2}}\left[\frac{q^{2}}{p^{2}}\left(\log \frac{\min \left(p^{2},(\vec{q}+\vec{p})^{2}\right)}{\Lambda^{2}}+\frac{\eta_{0}}{2}\right)+\frac{p^{2}}{q^{2}}\left(\log \frac{\min \left(q^{2},(\vec{q}+\vec{p})^{2}\right)}{\Lambda^{2}}+\frac{\eta_{0}}{2}\right)\right. \\
& \left.\left.-\frac{1}{2}\left(1+\vec{q} \cdot \vec{p}\left(\frac{1}{p^{2}}+\frac{1}{q^{2}}\right)\right)\left(\log \frac{\min \left(p^{2}, q^{2},(\vec{q}+\vec{p})^{2}\right)}{\Lambda^{2}}+\frac{\eta_{0}}{2}+\frac{\eta_{0}^{2}}{8}\right)\right]+\mathcal{O}\left(\frac{\Lambda^{2}}{p^{2}}\right)\right\}+(\vec{q} \rightarrow-\vec{q}) .
\end{aligned}
$$

No logarithms of $Q^{2}$ appear here, since the gluon distribution in this biased ensemble, Eq. (55), drops more rapidly than $1 / k^{4}$. The angular moments are

$$
\begin{aligned}
\left\langle e^{2 n i \phi}\right\rangle_{\text {bias }} \approx & \frac{\pi}{\left(N_{c}^{2}-1\right) A_{\perp} \Lambda^{2}} \frac{\Lambda^{2}}{\left|p^{2}-q^{2}\right|} \frac{q_{<}^{2 n}}{q_{>}^{2 n}} \\
& \times \frac{\left(\frac{q^{2}}{p^{2}}+\frac{p^{2}}{q^{2}}\right)\left(\log \frac{\min \left(p^{2}, q^{2}, \ell^{2}\right)}{\Lambda^{2}}+\frac{5 \eta_{0}}{2}+\frac{\eta_{0}^{2}}{8}\right)+4 \frac{q^{2}}{p^{2}} \log \frac{\min \left(p^{2}, \ell^{2}\right)}{\Lambda^{2}}+4 \frac{p^{2}}{q^{2}} \log \frac{\min \left(q^{2}, \ell^{2}\right)}{\Lambda^{2}}}{\left(\log \frac{p^{2}}{\Lambda^{2}}+\frac{\eta_{0}}{2}\right)\left(\log \frac{q^{2}}{\Lambda^{2}}+\frac{\eta_{0}}{2}\right)+\frac{2 \pi \eta_{0}^{2}}{3 \Lambda^{2} A_{\perp}\left(N_{c}^{2}-1\right)}} .
\end{aligned}
$$

\footnotetext{
${ }^{7}$ Note that the dependence on $n$ cancels in these ratios.
} 
To simplify this expression, we consider various parametric magnitudes for $\eta_{0}$. The case $\eta_{0} \lesssim 1$ is not very interesting, as it does not lead to rare configurations with a substantial increase in the number of gluons; we have $V_{\text {eff }} \propto$ $N_{c}^{2} A_{\perp} \Lambda^{2} \eta_{0}$ and $\Delta N_{g} \propto N_{c}^{2} A_{\perp} g^{4} \mu^{2} \eta_{0}$, independent of $Q^{2}$.

It is more interesting to let $\eta_{0} \sim \sqrt{\log \frac{Q^{2}}{\Lambda^{2}}}$, so that the amplitude of the shift of the gluon distribution and its transverse momentum cutoff are related. $\Delta N_{g}$ then increases in proportion to $\sqrt{\log \frac{Q^{2}}{\Lambda^{2}}}$, while $V_{\text {eff }} \propto \sqrt{\log \frac{Q^{2}}{\Lambda^{2}}} \log \left(\log \frac{Q^{2}}{\Lambda^{2}}\right)$. This means that high gluon multiplicities can be reached with much higher probability than for the bias considered in the previous section. Furthermore, the angular moments will increase with $\eta_{0}^{2}$ for any choice of $p^{2}$ or $q^{2}$. For example, when $p^{2} \approx q^{2}$,

$$
\frac{\left\langle e^{2 n i \phi}\right\rangle_{\text {bias }}}{\left\langle e^{2 n i \phi}\right\rangle_{\text {unb }}} \approx 1+\frac{1}{40} \frac{\eta_{0}^{2}}{\log \frac{\ell^{2}}{\Lambda^{2}}},
$$

and the correction increases like the square of $\Delta N_{g}$, i.e., proportional to $\log Q^{2}$.

We briefly comment on the case where the gluon distribution at the saddle point of the reweighted ensemble is shifted to

$$
\eta(k)=1+\eta_{0} \frac{k^{2}}{\Lambda^{2}} \Theta\left(Q^{2}-k^{2}\right) \Theta\left(k^{2}-\Lambda^{2}\right),
$$

again with $Q^{2} \gg \Lambda^{2}$. This leads to a very strong increase of $\left\langle k_{T}^{2}\right\rangle$ as compared to the unbiased ensemble. On the other hand, to have $V_{\text {eff }} \propto Q^{2}$ like in Sec. III B, rather than $V_{\text {eff }} \propto Q^{4}$, we must choose very small amplitude, $\eta_{0} \propto 1 / Q^{2}$. And then, $\Delta N_{g}$ asymptotes to a constant at large $Q^{2}$. In other words, these are rare configurations of the hadron where the excess mean squared transverse momentum of the gluons is large but their number excess is not. In this paper we restrict our discussion to cases corresponding to large $\Delta N_{g}$.

\section{Anisotropic $\boldsymbol{\eta}(\vec{k})$}

In this section, we explore anisotropic gluon distributions such that the average gluon distribution $X_{s}(k)$ in the unbiased ensemble is multiplied by $\eta(\vec{k})=1+\eta_{0} \frac{\Lambda^{2 a}}{k^{2 a}}(\hat{k} \cdot \hat{E})^{2 b} \Theta\left(Q^{2}-k^{2}\right) \Theta\left(k^{2}-\Lambda^{2}\right)$.

The vector $\hat{E}$ specifies an arbitrary direction in the transverse plane. Studying such anisotropic configurations has been suggested by Kovner and Lublinsky [24] (also see Ref. [25]). In their work, the anisotropy is due to fluctuations from configuration to configuration of the hadron or nucleus. In this scenario, after computing the angular correlator, $\left\langle e^{i n \phi}\right\rangle$, we would have to perform an average over the directions of $\hat{E}$. In what follows, we will instead consider the possibility that the $2 \mathrm{~d}$ rotational symmetry is explicitly broken due to an external bias (like a spin model in an external magnetic field), so that Eq. (62) represents the gluon distribution averaged over the reweighted ensemble. It is beyond the scope of the present paper to discuss specific phenomenological models for how such an anisotropic bias may arise in $p+p$ or $p+A$ collisions. Nevertheless, it is an interesting exercise to compute twoparticle correlations in an anisotropic ensemble, as this leads to new contributions to the angular moments.

Specifically, the disconnected diagram, $\mathcal{A}$, will now contribute $\phi$-dependent terms. From Eqs. (34), (36), we see that these terms only occur when $Q^{2} \sim p^{2}, q^{2}$, and that they will be proportional to $\eta_{0}^{2}\left[(\hat{p} \cdot \hat{E})^{2}(\hat{q} \cdot \hat{E})^{2}\right]^{b}$. Therefore, only moments less than or equal to $2 b$ will receive such contributions.

When $a=1$, the leading angular contribution to $\mathcal{A}$ is proportional to $\frac{\Lambda^{4}}{p^{4}} \log ^{2} \frac{p^{2}}{\Lambda^{2}}$, which is smaller than the angular contributions we saw in Sec. III B. Hence, we consider the case $a=0$ and $b=1$. We then have, for $\eta_{0} \ll 1, Q^{2} \gg \Lambda^{2}$,

$$
V_{\text {eff }} \approx \frac{3}{128 \pi} N_{c}^{2} A_{\perp} Q^{2} \eta_{0}^{2}
$$

and

$$
\Delta N_{g}=\frac{1}{16 \pi} N_{c}^{2} A_{\perp} g^{4} \mu^{2} \eta_{0} \log \frac{Q^{2}}{\Lambda^{2}} .
$$

The contribution from the disconnected diagram now becomes (for $Q^{2} \sim p^{2}, q^{2}$ )

$$
\mathcal{A}_{\text {bias }} \approx\left(N_{c}^{2}-1\right) A_{\perp} \Lambda^{2} \log \frac{p^{2}}{\Lambda^{2}} \log \frac{q^{2}}{\Lambda^{2}}\left[1+\frac{\eta_{0}}{4}+\frac{\eta_{0}^{2}}{2}(\hat{p} \cdot \hat{E})^{2}\right]\left[1+\frac{\eta_{0}}{4}+\frac{\eta_{0}^{2}}{2}(\hat{q} \cdot \hat{E})^{2}\right] .
$$

Because this is the contribution from the "disconnected diagram," and there is a constant $k$-independent boost of the gluon distribution relative to the unbiased ensemble, we recover the two DGLAP logarithms even in the anisotropic contribution. 
The anisotropic part of $\mathcal{B}$ at linear order in $\eta_{0}$ is ${ }^{8}$

$$
\begin{aligned}
\mathcal{B}_{\text {bias }}-\mathcal{B}_{\text {unb }} \approx & \frac{\pi \eta_{0}}{4} \frac{\Lambda^{2}}{(\vec{p}+\vec{q})^{2}}\left\{( 8 ( \hat { p } \cdot \hat { E } ) ^ { 2 } + 4 [ \hat { E } \cdot ( \widehat { p + q } ) ] ^ { 2 } + 2 ) \left[\frac{q^{2}}{p^{2}} \log \frac{\min \left(p^{2},(\vec{p}+\vec{q})^{2}\right)}{\Lambda^{2}}\right.\right. \\
& \left.-\frac{1}{8}\left(1+\vec{q} \cdot \vec{p}\left(\frac{1}{p^{2}}+\frac{1}{q^{2}}\right)\right) \log \frac{\min \left(p^{2}, q^{2},(\vec{p}+\vec{q})^{2}\right)}{\Lambda^{2}}\right]+\log \frac{\min \left(p^{2}, q^{2},(\vec{p}+\vec{q})^{2}\right)}{\Lambda^{2}} \\
& \left.\times\left[(\hat{p} \cdot \hat{q})^{2}[\hat{E} \cdot(\widehat{p+q})]^{2}+\frac{\hat{E} \cdot \vec{p}}{p^{2}} \hat{E} \cdot\left(\vec{q}-\frac{\vec{p} \cdot \vec{q}}{p^{2}}(\vec{p}+\vec{q})\right)\right]+(\vec{p} \leftrightarrow \vec{q})\right\}+(\vec{q} \rightarrow-\vec{q})
\end{aligned}
$$

(vectors with a hat denote unit vectors). Equations (65), (66) both give rise to nonzero odd angular moments. Integrating $\mathcal{A}_{\text {bias }} \cos n \phi$ over $\phi$, we get

$$
\int \mathrm{d} \phi \cos (n \phi) \mathcal{A}_{\text {bias }} \approx\left(N_{c}^{2}-1\right) A_{\perp} \Lambda^{2} \log \frac{p^{2}}{\Lambda^{2}} \log \frac{q^{2}}{\Lambda^{2}} \frac{\pi}{32} \begin{cases}\left(16 \eta_{0}+8 \eta_{0}^{2}\right) \cos \left(\psi-2 \phi_{E}\right) & n=1 \\ \eta_{0}^{2} & n=2, \\ 0 & n \geq 2\end{cases}
$$

where $\psi$ is the "center of mass angle" (the average of the angles made by $\vec{p}$ and $\vec{q}$ ) and $\phi_{E}$ is the angle made by $\hat{E}$. If the anisotropy is due to fluctuations, and we average over the direction $\phi_{E}$ of $\hat{E}$, then only the contribution to the $n=2$ elliptic moment is nonzero. On the other hand, for an external bias with fixed direction, there is a nonzero $n=1$ moment when the average azimuthal angle of $\vec{p}$ and $\vec{q}$ is not equal to that of $\hat{E}$ plus $45^{\circ}$.

In the following, we focus on the elliptical anisotropy. By comparing Eq. (65) with Eq. (66), we see that, if we choose $1 \gg \eta_{0} \gg \frac{\Lambda^{2}}{Q^{2}}\left(\log \frac{Q^{2}}{\Lambda^{2}}\right)^{-1}$, then the main contribution to the angular moments will be from $\mathcal{A}_{\text {bias }}$, with $\mathcal{B}_{\text {bias }}$ a small correction. The elliptical anisotropy is then

$$
\left\langle e^{2 i \phi}\right\rangle_{\mathrm{bias}} \approx\left\langle e^{2 i \phi}\right\rangle_{\mathrm{unb}}+\frac{\eta_{0}^{2}}{64},
$$

which increases with increasing $\eta_{0}$. The remarkable aspect of this expression is that the correction due to the bias is independent of $p$ and $q$, and that it will dominate the azimuthal correlation for sufficiently large $p^{2}, q^{2},\left|p^{2}-q^{2}\right|$.

\section{SUMMARY AND CONCLUSION}

Imposing a bias on the functional integral over small- $x$ gluon distributions in a hadron (or photon or nucleus) modifies the expectation value of the gluon distribution, as well as statistical fluctuations about it. One may bias with respect to the multiplicity of gluons, their average squared transverse momentum, their transverse energy, or, more generally, towards a specific modification of their

\footnotetext{
${ }^{8} \mathrm{We}$ do not include the contribution to $\mathcal{B}$ of order $\eta_{0}^{2}$ because it is a subleading contribution to the part of the cross section of order $\eta_{0}^{2}$, and because we restrict to the elliptic anisotropy in this section.
}

distribution over transverse momentum. Another example is $p+p$ collision events with a hard jet or a $Z$-boson. Each of these biases produces a distinct "distortion" of the average gluon distribution. The modification of various observables under such biases provides, in principle, a test of our understanding of high-energy QCD, a fact well known to and appreciated by developers of "event generators" for high-energy collisions. Our specific focus here (in continuation of prior work in Refs. $[4,6]$ ) is on relating the bias to reweighting of the functional integral over the effective action for small- $x$ gluons.

In the present paper, we analyzed the effect of some simple model biases on the two-gluon transverse momentum spectrum, as well as their azimuthal correlations, in a high-energy collision. We found interesting differences between the biased ensembles that were studied. For example, when the bias boosts the gluon density uniformly by a constant factor, for all $k$ up to the hard momenta $p$ and $q$ of the produced gluons, there is no effect on the azimuthal moments. Such a modification, however, has essentially infinite action, and probability zero. On the other hand, if one increases the density of gluons uniformly by a constant factor, but only over transverse momentum squared $\ll p^{2}, q^{2},(\vec{p} \pm \vec{q})^{2}$, the azimuthal moments are affected by the bias. This is due to the fact that the Lipatov process involves fusion of two gluons, one with $k$ much less than $p$, $q$ and the other with $k$ comparable to $p, q$. We find that the elliptic angular moment, $\langle\exp (2 i \phi)\rangle$, increases with the gluon number excess $\Delta N_{g}$ when $\vec{p}$ and $\vec{q}$ have comparable magnitude, but decreases with $\Delta N_{g}$ when $p \gg q$.

Another example of at least theoretical interest is that of a bias that introduces a preferred direction in the transverse impact parameter plane, inducing an anisotropy of the gluon distribution. Such a bias gives rise to an angular dependence even of the disconnected (in terms of color flow) diagram for double gluon production. Hence, the 
leading dependence of the cross section on the azimuthal angle $\phi$ between $\vec{p}$ and $\vec{q}$ emerges at leading power in $N_{c}$, and comes with the same DGLAP $\log$ arithms $\log p^{2} \log q^{2}$ as the diagrams for uncorrelated production in an unbiased ensemble.

Our analysis could be continued by looking at more complicated biases and how they would reweight the functional integral over gluon distributions. In particular, a very important step would be to develop a more direct connection between the reweighting functional $b[X]$ used here and specific event ensembles that one might be able to construct in practice, either from experiment or from an event generator.

\section{ACKNOWLEDGMENTS}

I gratefully acknowledge support by the DOE Office of Nuclear Physics through Grant No. DE-FG02-09ER41620; and from The City University of New York through the PSC-CUNY Research Grant No. 62098-0050. I thank A. Dumitru for numerous useful discussions and suggestions and for help with the preparation of the manuscript. I also thank V. Skokov for comments on the draft.
[1] H. Weigert, Prog. Part. Nucl. Phys. 55, 461 (2005).

[2] J. P. Blaizot, E. Iancu, and H. Weigert, Nucl. Phys. A713, 441 (2003); A. Kovner, Acta Phys. Pol. B 36, 3551 (2005).

[3] L. D. McLerran and R. Venugopalan, Phys. Rev. D 49, 2233 (1994); 49, 3352 (1994); Yu. V. Kovchegov, Phys. Rev. D 54, 5463 (1996).

[4] A. Dumitru and V. Skokov, Phys. Rev. D 96, 056029 (2017).

[5] R. Baier, Y. L. Dokshitzer, A. H. Mueller, S. Peigne, and D. Schiff, Nucl. Phys. B484, 265 (1997).

[6] A. Dumitru, G. Kapilevich, and V. Skokov, Nucl. Phys. A974, 106 (2018).

[7] A. Dumitru, F. Gelis, L. McLerran, and R. Venugopalan, Nucl. Phys. A810, 91 (2008); A. Dumitru, K. Dusling, F. Gelis, J. Jalilian-Marian, T. Lappi, and R. Venugopalan, Phys. Lett. B 697, 21 (2011).

[8] K. Dusling, F. Gelis, T. Lappi, and R. Venugopalan, Nucl. Phys. A836, 159 (2010).

[9] Y. V. Kovchegov and D. E. Wertepny, Nucl. Phys. A906, 50 (2013); A925, 254 (2014).

[10] T. Lappi, B. Schenke, S. Schlichting, and R. Venugopalan, J. High Energy Phys. 01 (2016) 061.

[11] T. Altinoluk, N. Armesto, and D. E. Wertepny, J. High Energy Phys. 05 (2018) 207; T. Altinoluk, N. Armesto, A. Kovner, and M. Lublinsky, Eur. Phys. J. C 78, 702 (2018).

[12] A. Kovner and M. Lublinsky, Int. J. Mod. Phys. E 22, 1330001 (2013).

[13] S. Schlichting and P. Tribedy, Adv. High Energy Phys. 2016, 8460349 (2016).

[14] K. Dusling and R. Venugopalan, Phys. Rev. Lett. 108, 262001 (2012); Phys. Rev. D 87, 054014 (2013).
[15] M. Mace, V. V. Skokov, P. Tribedy, and R. Venugopalan, Phys. Lett. B 788, 161 (2019).

[16] B. Blok, C. D. Jäkel, M. Strikman, and U. A. Wiedemann, J. High Energy Phys. 12 (2017) 074; B. Blok and U. A. Wiedemann, Phys. Lett. B 795, 259 (2019).

[17] B. Schenke, C. Shen, and P. Tribedy, arXiv:1908.06212.

[18] L. V. Gribov, E. M. Levin, and M. G. Ryskin, Phys. Rep. 100, 1 (1983).

[19] T. Altinoluk and A. Dumitru, Phys. Rev. D 94, 074032 (2016); P. Agostini, T. Altinoluk, and N. Armesto, Eur. Phys. J. C 79, 600 (2019).

[20] T. Altinoluk, N. Armesto, G. Beuf, A. Kovner, and M. Lublinsky, Phys. Lett. B 752, 113 (2016).

[21] T. Altinoluk, N. Armesto, G. Beuf, A. Kovner, and M. Lublinsky, Phys. Lett. B 751, 448 (2015).

[22] V. N. Gribov and L. N. Lipatov, Yad. Fiz. 15, 781 (1972) [Sov. J. Nucl. Phys. 15, 438 (1972)]; Yad. Fiz. 15, 1218 (1972) [Sov. J. Nucl. Phys. 15, 675 (1972)]; G. Altarelli and G. Parisi, Nucl. Phys. B126, 298 (1977); Yu. L. Dokshitzer, Zh. Eksp. Teor. Fiz. 73, 1216 (1977) [Sov. Phys. JETP 46, 641 (1977)]; Yu. L. Dokshitzer, D. Diakonov, and S. I. Troian, Phys. Rep. 58, 269 (1980).

[23] E. A. Kuraev, L. N. Lipatov, and V. S. Fadin, Zh. Eksp. Teor. Fiz. 72, 377 (1977) [Sov. Phys. JETP 45, 199 (1977)]; I. I. Balitsky and L. N. Lipatov, Yad. Fiz. 28, 1597 (1978) [Sov. J. Nucl. Phys. 28, 822 (1978)].

[24] A. Kovner and M. Lublinsky, Phys. Rev. D 84, 094011 (2011).

[25] A. Dumitru and V. Skokov, Phys. Rev. D 91, 074006 (2015). 